\title{
Higher adherence to Mediterranean Diet is associated with improved semen parameters: A systematic review and meta-analysis of observational studies
}

\section{Abstract}

Semen quality is a key indicator of male fecundity and therefore is closely related to male fertility. In recent decades a significant decrease of semen parameters was observed worldwide. Except for environmental pollution, low physical activity, body weight and comorbidities, unhealthy diet was linked to semen quality decline. Mediterranean Diet (MedDiet) due to its anti-oxidative and anti-inflammatory properties, is considered as beneficial for semen. However, results of existing studies are inconclusive. The aim of this review was to pool associations between adherence to the MedDiet and semen quality parameters in observational studies. Electronic databases including PubMed, Embase and Scopus were searched until April 2019 with no restriction to language or time. Studies were eligible for inclusion if they filled following criteria: (1) observational design (retrospective or prospective), (2) adherence to MedDiet assessed using score or data-driven method and (3) semen quality parameters (total motility, progressive motility, total count, concentration or volume) as outcomes. Mean differences or odds ratios of abnormal semen parameters between the highest and lowest quintiles of MedDiet were converted to standardized mean differences (SMD). Random effects models with inverse variance method were used to pool differences. Heterogeneity was assessed using the Cochrane Q test and $\mathrm{I}^{2}$ statistic with value $>50 \%$ indicating substantial heterogeneity. Five cross-sectional studies extracted from 840 articles, met the eligibility criteria and were included in the meta-analysis. Higher adherence to MedDiet compared to lower adherence was associated with increased total (SMD $=0.59$ $\left.95 \% \mathrm{CI}: 0.12-1.06, \mathrm{I}^{2}=80 \%, \mathrm{p}_{\text {heterogenity }}<0.01, \mathrm{n}=4\right)$ and progressive $\left(\mathrm{SMD}=0.5395 \% \mathrm{CI}: 0.16-0.91, \mathrm{I}^{2}=0 \%, \mathrm{p}_{\text {heterogenity }}=0.94\right.$, $\mathrm{n}=2)$ sperm motility. No associations were observed between adherence to MedDiet and total sperm count (SMD $=0.1495 \% \mathrm{CI}$ : $-0.20-0.48, \mathrm{I}^{2}=70 \%, \mathrm{p}_{\text {heterogenity }}<0.01, \mathrm{n}=5$ ), concentration $\left(\mathrm{SMD}=0.2295 \% \mathrm{CI}:-0.04-0.47, \mathrm{I}^{2}=48 \%, \mathrm{p}_{\text {heterogenity }}=0.10, \mathrm{n}=5\right.$ ), volume $\left(\mathrm{SMD}=0.0295 \% \mathrm{CI}:-0.20-0.25, \mathrm{I}^{2}=21 \%\right.$, pheterogenity $\left.=0.28, \mathrm{n}=5\right)$, as well as morphology $(\mathrm{SMD}=-0.1095 \% \mathrm{CI}:-0.36$ $0.16, \mathrm{I}^{2}=38 \%$, pheterogenity $\left.=0.18, \mathrm{n}=4\right)$. The results suggest that higher adherence to the MedDiet may have a protective effect on semen quality, probably by increased intake of antioxidant-rich products. Limitations of findings include substantial between-study heterogeneity and use of cross-sectional design. Beneficial effects of the MedDiet on semen quality have to be confirmed in further prospective studies.

\section{Conflict of Interest}

There is no conflict of interest 